\title{
CAMBIOS EN LA OCUPACIÓN DE LOS ADULTOS MAYORES RECIENTEMENTE JUBILADOS
}

\author{
CHANGES IN THE OCCUPATION OF RECENTLY RETIRED OLDER ADULTS
}

\author{
Allan A., Natalia', Wachholtz M., Daniela² y Valdés R, Alicia ${ }^{3}$
}

\begin{abstract}
RESUMEN
El presente estudio tiene como objetivo comprender cómo el proceso de jubilación influye en el desempeño ocupacional de los adultos mayores recientemente jubilados. Para ello, se indaga sobre conceptos relacionados con la jubilación y cómo se entiende ésta desde las distintas disciplinas. A su vez, es necesario comprender el concepto de ocupación para poder asociar el desempeño ocupacional que adoptan los jubilados en las diversas áreas de desempeño (Actividades de la Vida Diaria, Productividad y Esparcimiento) desde el enfoque del Modelo de Ocupación Humana. Para identificar esta correlación fueron efectuadas entrevistas en profundidad a participantes de ambos sexos, que tuvieran como máximo tres años de retiro laboral. Las entrevistas fueron diseñadas utilizando un instrumento de evaluación creado a partir del opHI-II. Los resultados obtenidos permiten observar que el proceso de jubilación no provocaría un desequilibrio ocupacional y que estos individuos lograrían adaptarse a dicho proceso, creando una nueva estructura en sus vidas y permitiéndoles alcanzar el nivel de competencia en las distintas ocupaciones en las que se involucran.
\end{abstract}

\section{PALABRAS CLAVE}

Ocupación, Disfunción ocupacional, Jubilación, Modelo de ocupación humana

\begin{abstract}
The objective of the present study is to understand how the retirement process influences the occupational performance of the recently retired elders. To achieve this, retirement related concepts are studied and analyzed from the point of view of different disciplines. The comprehension of the "occupation" concept is also needed, to be able to associate the occupational performance that retired elders adopt in different areas (Activities of Daily Living, Productivity and Play Activities) with the approach given by the Model of Human Occupation. To be able to identify this relation, several interviews were done to participants of both sexes and that were retired for no more than three years. The interviews were designed using an evaluation instrument created from the OPHI-II. The results of this study show that the retirement process does not cause an occupational unbalance, and that these individuals are able to adapt to the process, creating a new structure in their lives and allowing them to reach a competent level in the different occupations in which they are involved.
\end{abstract}

\section{KEYWORDS}

Occupation, Occupational Dysfunction, Retirement, Human Occupation Model

Terapeuta Ocupacional, Licenciada en Ciencias de la Ocupación Humana. Universidad de Chile.

2 Terapeuta Ocupacional, Licenciada en Ciencias de la Ocupación Humana. Universidad de Chile.

3 Terapeuta Ocupacional, Licenciada en Ciencias de la Ocupación Humana. Universidad de Chile. Master en Integración de Personas con Discapacidad. Universidad de Salamanca, España. 


\section{INTRODUCCIÓN}

El siguiente estudio pretende brindar mayores conocimientos acerca del impacto que el proceso de jubilación tiene en la ocupación de los adultos mayores, pudiendo así colaborar con información relevante a considerar en la formulación y elaboración de Políticas Públicas, Sociales y de Salud. Además, se busca entregar conocimientos en profundidad acerca del tema para establecer las bases de futuras investigaciones y entregar información de acuerdo a los distintos sexos, en relación al significado que se le otorga a la ocupación después de jubilar.

Por último, se pretende contribuir con nuevos conocimientos que sirvan de aporte a la Ciencia de la Ocupación y además describir los cambios que se producen en el desempeño ocupacional, en las actividades de la vida diaria, productivas, de esparcimiento y de ocio de los adultos mayores recientemente jubilados.

\section{El proceso de jubilación}

Existen distintos enfoques conceptuales a la hora de definir el proceso de jubilación, como el punto de vista económico, donde la persona entra en el proceso de jubilación cuando cumple con la etapa laboral productiva, o el punto de vista social, en el cual conlleva a la pérdida del rol productivo, siendo en ambos casos considerado como un derecho social acumulado luego de una vida de trabajo.

Por otro lado, la jubilación, entendida como etapa del ciclo vital, implica dejar el trabajo cuando ya no se es capaz de cumplir cabalmente las obligaciones que éste impone, debido a la natural declinación de las capacidades físicas y psicológicas asociadas al proceso de envejecimiento. Este último enfoque es el eje principal del presente estudio.

\section{La jubilación como problema social}

La jubilación, proceso que debiera ser visto como un logro de la humanidad, tiende a ser percibido en términos de los problemas que genera: como el costo que significa la mantención y el cuidado de un creciente número de individuos económicamente dependientes, que sin aportar directamente a una generación de producto requieren utilizar parte de éstos ${ }^{(5)}$.

Por otra parte $-y$ desde el punto de vista de la literatura revisada-el proceso de jubilación en nuestra sociedad traería consigo una serie de consecuencias negativas: en el aspecto económico, los ingresos significativamente menores; un deterioro en las relaciones sociales, por la pérdida de contacto con los compañeros de trabajo y la pérdida de estatus como individuo activo, sumada a la desaparición de seres queridos y amistades que fallecen; un deterioro en la salud, debido a la ruptura y al cambio súbito de las rutinas que entrega la vida laboral.

Una problemática importante de esta etapa de vida, es la pérdida del rol social asignado y aceptado, que luego de jubilar, se centra en actividades de ocio y tiempo libre, siendo éstas consideradas un estado pasivo del individuo a nivel social productivo y no aceptadas por las personas aún competentes.

\section{Concepto de Ocupación}

Para comprender la transformación que ocurre en las ocupaciones de las personas jubiladas, se debe realizar un análisis de las variables que se conjugan en el proceso de retiro laboral y analizarlas desde el punto de vista del Modelo de Ocupación Humana. Los individuos experimentan y expresan significado en sus vidas por medio de sus ocupaciones. Las ocupaciones no sólo tienen significado por si mismas sino en relación con otras ocupaciones y en la completa organización de la vida. En este estudio se considera el concepto de Ocupación como parte fundamental del quehacer del ser humano y se consideran los tres tipos o áreas de ocupación definidos según la Asociación Americana de Terapia Ocupacional:

- Actividades de la Vida Diaria: abarcan el autocuidado, las tareas domésticas, el mantenimiento del espacio vital, el manejo y mantenimiento de los recursos económicos y aquellas conductas adecuadas para el acceso a los distintos recursos del entorno (compras, desplazamientos, viajes).

- Actividades Productivas: actividades de trabajo remuneradas o no, es decir, aquellas que proporcionan un servicio a la sociedad (a un miembro, a varios o al conjunto). 
- Actividades de Esparcimiento: actividades llevadas a cabo por propio gusto, como celebrar, participar en juegos, deportes, o cultivar pasatiempos.

El ideal ocupacional se ve reflejado por un equilibrio, el cual corresponde a una distribución balanceada de tiempo entre actividades de la vida diaria, productivas, esparcimiento y descanso. Este equilibrio se manifiesta también cuando las habilidades coinciden con la ocupación, el deseo y las necesidades del individuo y de su ambiente.

\section{Modelo de Ocupación Humana}

El estudio se encuentra enmarcado dentro del Modelo de Ocupación Humana (мон), ya que este modelo, propio de la Terapia Ocupacional, interpreta a los seres humanos como sistemas abiertos y dinámicos, resaltando el "Comportamiento Ocupacional" como un proceso organizador. (12)

El $\mathrm{MOH}$ conceptualiza a la persona como un sistema compuesto por tres subsistemas: Volición, Habituación y Capacidad de Desempeño.

El propósito del Subsistema Volición es seleccionar el Comportamiento Ocupacional. El Subsistema Habituación, sirve a la organización del Comportamiento Ocupacional en patrones o rutinas. Finalmente, el Subsistema Capacidad de Desempeño hace posible la realización hábil de las ocupaciones.

El término Volición ${ }^{(13)}$ se refiere a voluntad o elección conciente, por lo que se relaciona estrechamente con el concepto de elecciones ocupacionales, las cuales son decisiones cotidianas respecto a aquellas actividades ocupacionales que la persona decide realizar en un momento determinado, es decir, son decisiones deliberadas de entrar y salir de actividades ocupacionales. Al involucrarse en este tipo de actividad, la persona asume un rol ocupacional que debe desempeñary a través del cual se emprende un proyecto personal.

El Subsistema de Habituación (14) permite comprender por qué las personas repiten patrones familiares de comportamiento. La Habituación depende de un circuito de tendencias apreciativas organizadas, las cuales permiten al ser humano improvisar modos similares de comportarse en circunstancias similares a lo largo del tiempo, sin deliberación o atención.

El subsistema Capacidad de Desempeño ${ }^{\left({ }^{15}\right)}$ es un conjunto de habilidades que son utilizadas en la elaboración de un comportamiento con propósito y cumple con la organización de los constituyentes físicos y mentales.

$\mathrm{El} \mathrm{MOH}$, también incluye a los contextos ambientales $\underline{(16)}$ del comportamiento ocupacional, que son una combinación de espacios, objetos, formas ocupacionales y/o grupos sociales coherentes que constituyen un contexto significativo para el desempeño. Dentro de estos están: el hogar, vecindario, escuela, lugar de trabajo y lugares de reunión y recreación.

\section{La jubilación como transición ocupacional}

El proceso de jubilación implica una transición de un estado ocupacional a otro, llevando al despliegue de distintos mecanismos de adaptación.

El MOH señala que los ciclos adaptativos conducen a la Función Ocupacional, que se da cuando el individuo satisface las necesidades propias y las expectativas del entorno. Los ciclos desadaptativos generan un estado de Disfunción Ocupacional, que se da cuando las expectativas sociales y las necesidades propias de exploración y dominio no son satisfechas. El modelo propone un continuo que va del logro, la competencia y la exploración como elementos de funcionamiento adaptativo, a la ineficacia, incompetencia e impotencia dentro del funcionamiento desadaptativo.

Según G. Kielhofner, el ser competente es un motivo universal, energizado por el deseo de ser reconocido por nuestros pares como una persona adecuada. El ser competente significa adecuarse a las demandas de una circunstancia o tarea. La meta u objetivo de la competencia, es ser capaz de relacionarse de manera efectiva con el ambiente y mejorar o moldearse uno mismo y sus propias habilidades para el desempeño de acuerdo con el feedback recibido.

Por lo anteriormente descrito, la jubilación podría ser considerada una situación de "riesgo ocupacional", en el sentido de que podría conllevar a una posible disfunción ocupacional. 


\section{Disfunción Ocupacional}

Las disfunciones ocupacionales ${ }^{(24)}$ resultan de la interrelación entre factores biológicos, psicológicos y ecológicos (G. Kielhofner). Una disfunción en el desempeño ocupacional ocurre cuando al individuo, con capacidad limitada, le falta confianza y no sabe como resolver el problema efectivamente para compensar sus limitaciones, encontrando barreras físicas y sociales.

La palabra "disfunción" se utiliza para indicar una incapacidad crónica o temporal para manejar y participar en los roles, relaciones y ocupaciones esperadas de una persona de similar edad y cultura. Las causas de tal incapacidad pueden incluir factores físicos, sociales, psicológicos o ambientales.

Todo cambio requiere de ajustes, transformaciones de hábitos y formas de hacer, como una forma de adaptación a la nueva realidad. Es sabido que los individuos llevan consigo a la etapa de la vejez, los mismos estilos de reaccionar ante las situaciones que han desarrollado a través de toda su vida.

\section{Evidencias acerca del impacto de la jubilación en el desempeño ocupacional}

Considerando los planteamientos presentados desde el punto de vista de la Ocupación Humana, el hombre es por sobre todo un ser biopsicosocial, inmerso en un medio familiar que desarrolla una función laboral y se desenvuelve en un medio social.

Del estudio que realiza Capetillo y Flores en el año $1990^{(15)}$, se desprende que jubilar significa mucho más que dejar de desempeñar una determinada actividad laboral. También significa una pérdida de una fuente de gratificaciones de gran importancia para la vida del hombre, pues el trabajo tiene para la persona numerosos significados.

El jubilado debe establecer un nuevo equilibrio en su ritmo vital, y si se poseen intereses y se han explorado alternativas, existen buenas posibilidades para ocupar el tiempo de manera gratificante y significativa.

Aragoneses ${ }^{(5)}$ sostiene que el hombre que jubila inicia este período con sentimientos ambivalentes ante la pérdida del trabajo. Por una parte, experimenta satisfacción al no estar sometido a la dureza de la competencia actual, mientras por otro lado se produce una sensación de vacío y de inutilidad que lo lleva a realizar labores por las que antes no se interesaba. Sostiene además que las personas, al pasar por la etapa de jubilación, suelen distraerse más bien en actividades de tipo recreativo como clubes, organizaciones políticas o paseando en parques.

Analizado desde el aspecto familiar, Domínguez (19) define que el jubilado siente que declina su capacidad para desempeñarse como padre, compañero o proveedor. Coincide esto con otros autores, al señalar que el anciano no ejerce roles alternativos, salvo los que le corresponden a su condición de abuelo y que suelen aumentar las tensiones entre sus hijos por los problemas que genera su cuidado, tanto de orden económico como de atención personal.

En el aspecto social, el incumplimiento de la autorrealización de los jubilados y la falta de tareas especificadas culturalmente, conllevaría a la dificultad de saber en qué concentrar y en qué redirigir los esfuerzos, de modo de actualizar las propias potencialidades, las que pudiesen haberse desarrollado con anterioridad. Es aquí donde no se ha considerado la importancia que pueda tener la realización de otras actividades ajenas al trabajo.

La Teoría de Actividad elaborada por George Maddos en el año 1974, plantea que: "Mientras mayor número de roles o actividades ocupacionales posea la persona, según entra en la edad avanzada, mejor resistirá los efectos desmoralizantes de salir de los roles obligatorios a los que ordinariamente se les da prioridad en la vida adulta"(5).

La etapa de prejubilación, según el Psiquiatra Francisco Alonso Fernández ${ }^{(11)}$, es un período en el cual la persona no sabe cómo organizar el tiempo libre del cual dispone, siendo esto, un factor predisponente de problemas $y$ trastornos mentales.

En el estudio realizado en Chile por Carmen Barros ${ }^{(21)}$, mediante una muestra de 34 sujetos mayores de 65 años, señala que, en general una minoría de los adultos mayores estudiados sigue realizando alguna actividad económica. Tres de cada diez entrevistados evalúan positivamente su retiro laboral, en la medida en que han conseguido "amoldarse". Asimismo, los jubilados que 
califican su anterior trabajo como insatisfactorio, refieren que la jubilación representa un hecho positivo por la liberación de obligaciones desagradables (e.g cumplir horarios, asumir responsabilidades no deseadas).

El hecho de disponer de mayor cantidad de tiempo libre, según señalan los entrevistados, produce una cierta desestructuración temporal de las actividades diarias, ya que la jornada laboral era considerada como eje alrededor del cual se organizaban dichas actividades; siendo esta actividad la que permitía que el uso del tiempo tuviera un propósito, y que durante su transcurso existiera la oportunidad de interactuar con otros.

En la misma encuesta, tres de cada diez perciben el acontecimiento de modo negativo. El grupo que evalúa negativamente su retiro laboral señala que los hace sentirse desorientados y desvalorados, mientras que para otros, lo más importante es la disminución de ingresos, que incide en una limitación de la independencia y posibilidad de realización personal.

Cuatro de cada diez perciben el retiro como esperable y natural; considerando la jubilación como un cambio inevitable al que se deben adecuar.

En el ámbito de las actividades de la vida diaria, la mitad de los entrevistados del estudio de C. Barros (21), dicen haber experimentado una disminución en sus actividades domésticas (jardín y reparaciones varias en el caso de los hombres y cuidado de otros o cocinar en las mujeres). Las razones son atribuidas a la reducción en el número de habitantes del hogar, el traslado a residencias más pequeñas y la necesidad de adecuar la intensidad de las tareas domésticas a la disminución de las capacidades físicas. Esta disminución de actividades es valorada positivamente (por no ser gratas), y la minoría que ve en este hecho algo negativo es porque lo asocia al desmedro físico. Como contrapartida, un porcentaje menor señala haber comenzado a realizar labores domésticas en esta etapa; los varones, ya que están más horas en casa, y las mujeres, por ver disminuidos sus ingresos y en consecuencia por incapacidad de pagar el servicio doméstico.

Respecto de las actividades sociales, la mayoría absoluta manifiesta haber experimentado cambios, disminuyendo sus actividades (enjuiciando esta merma como normal, ya que en esta etapa es necesaria mayor tranquilidad, sin agitaciones y cuidando de la salud). De entre las causas referidas se señalan; la pérdida de amistades por enfermedad, muerte o baja de ingresos y el miedo a salir de noche. Además, la jubilación implicaría la ausencia de un conjunto de personas con las que se interactuaba a diario. En consecuencia, el retiro marca "un corte" en las posibilidades de interacción social. Por otra parte, y siguiendo el citado estudio de C. Barros (221), una minoría aumentó sus actividades sociales (valorándolo positivamente como fuente de enriquecimiento personal) o no experimentó cambios significativos.

Los sentimientos asociados al hecho de jubilar varían según la interpretación dada a este hecho. Para quienes jubilar fue negativo, el sentimiento predominante fue el dolor, desconcierto y sensación de inutilidad. En otros casos, fue de una tranquila resignación. La modalidad más usada para encarar esta nueva etapa fue la búsqueda de actividades alternativas (incorporación a grupos, cursos o actividades laborales independientes).

En el estudio realizado por Jonsson (22) en personas en transición ocupacional del trabajo a la jubilación, se delinean cuatro temas emergentes: una nueva estructura temporal, entrando en un ritmo mas lento; cambios en el significado de las ocupaciones; la paradoja de ser el "dueño" del propio tiempo; y la mantención de la estabilidad como el deseo principal para el futuro.

En relación al cambio en el significado de las ocupaciones, los hallazgos principales de esta investigación destacan que algunas ocupaciones son "redescubiertas" cuando se pierden las viejas estructuras de vida. Es así como las tareas domésticas y los roles conservados son enfrentados de modo distinto, produciéndose, en la mayoría de los casos, una mayor valoración y dedicación a éstos. De este modo, el autor concluye que las ocupaciones no poseen significado en sí mismas, sino en relación a las otras ocupaciones y a la organización total de la vida.

\section{Metodología}

El estudio se centra en adultos mayores recientemente jubilados, con un mínimo de un año y máximo de tres años de retiro laboral, de ambos sexos, residentes en la Región Metropolitana del ciudad de Santiago de Chile, que fueron contactados de manera individual. El tamaño de la muestra fue de 15 adultos mayores; 11 mujeres 
y 4 hombres. Los criterios de inclusión y exclusión utilizados son:

- Personas que accedan de manera voluntaria a una entrevista personalizada.

- Su capacidad de comunicación se encuentre indemne.

- Residentes de la Región Metropolitana de Santiago.

- Que presenten como mínimo un año y máximo tres años de retiro laboral.

Para recoger la información se utilizó la Entrevista en Profundidad, como un instrumento adecuado para la finalidad de conocer con mayor profundidad aspectos subjetivos de la vivencia de esta etapa en la vida de las personas.

La Pauta de Entrevista se construye en base al Modelo de Ocupación Humana, específicamente al Instrumento de Evaluación OPHI-II, y a las Áreas de Desempeño Ocupacional.
Las entrevistas son grabadas y posteriormente transcritas para asegurar mayor confiabilidad en la recolección de los datos y facilitar el proceso de análisis de la información ${ }^{(8)}$. Las categorías de análisis definidas están descritas en la Tabla I.

\section{RESULTADOS}

\section{Resultados en Hombres}

- La mayoría percibe mayor libertad y tiempo para realizar todas sus actividades de manera más relajada, con menos presión y menos stress, ya que no deben cumplir con las obligaciones que les imponía el trabajo.

- Existe un reemplazo de la estructura de trabajo por actividades de tiempo libre, donde éstas son realizadas en el horario que era destinado al trabajo.

TABLA 1 CATEGORÍAS DE ANÁLISIS

\begin{tabular}{|c|c|}
\hline Análisis de Hombres & Análisis de Mujeres \\
\hline Actividades de la vida diaria & Actividades de la vida diaria \\
\hline Roles & Roles \\
\hline Rutinas & Rutinas \\
\hline Contextos Ambientales & Contextos Ambientales \\
\hline Actividades de la vida diaria & Actividades de la vida diaria \\
\hline Roles & Roles \\
\hline Rutinas & Rutinas \\
\hline Situación Actual & Situación Actual \\
\hline Percepción del proceso & Percepción del proceso \\
\hline Actividades de esparciamiento y ocio & \multirow{5}{*}{$\begin{array}{l}\text { Actividades de esparciamiento y ocio } \\
\text { Roles } \\
\text { Rutinas } \\
\text { Situación Actual } \\
\text { Percepción del procesoPercepción futura }\end{array}$} \\
\hline Roles & \\
\hline Rutinas & \\
\hline Situación Actual & \\
\hline Percepción futura & \\
\hline
\end{tabular}


- Existe una percepción de cambio en la calidad de vida, que no puede ser calificado como positivo ni negativo. Los entrevistados no realizan una comparación entre la calidad de vida que tenían antes de jubilar con la que tienen en la actualidad. Asimismo, refieren que el rol de trabajador no es determinante en su calidad de vida.

- La mayoría refiere una actitud madura y reflexiva respecto de la vida, sin darle tanto valor a lo material sino más bien al crecimiento personal (estudiar, adquirir nuevos conocimientos, realizar voluntariado, hacer pequeños viajes, etc.)

- La mayoría presenta una pérdida de contacto con sus compañeros de trabajo, ya que no todos jubilan al mismo tiempo. Esto hace que el tiempo que ellos tienen disponible debe ser coordinado con la disponibilidad que poseen las amistades que aún se encuentran trabajando.

- La mitad de la muestra, si tuviera la posibilidad de volver a trabajar no lo haría. La única persona que desea volver a trabajar no es por necesidad económica, sino más bien por la necesidad de sentirse competente, ya que los roles asociados al período de jubilación no le provocan satisfacción personal.

- La mayoría no se siente limitado físicamente ni económicamente para realizar algún tipo de actividad en la actualidad. La jubilación tampoco aparece como un motivo limitante en las actividades que decidan realizar.

- La gran mayoría está satisfecho y encantado con su situación de jubilado, lo cual se contradice con el significado que se le atribuye socialmente a este proceso.

- Tres de los cuatro entrevistados percibe la finitud de la vida y del tiempo disponible que le queda por vivir, el cual es considerado dentro de su actuar. Esto se expresa en una proyección a futuro limitada en el tiempo que no supera más allá de un año.

- La mitad hace alusión a que los dos períodos no son comparables, atribuyéndole a cada uno distintas connotaciones, pero destacando el período de jubilado como mejor por la mayor disponibilidad de tiempo y la menor cantidad de obligaciones impuestas.

- Uno de los entrevistados refiere que no presentaba planes concretos para realizar cuando estuviese jubilado y atribuye que la dificultad de adaptación al proceso estaría dada por la falta de definición de metas y planes futuros. Sin embargo, los tres res- tantes no sienten la necesidad de emprender cosas nuevas aún cuando se sienten capaces de realizarlo. - La mayoría, refiere un equilibrio ocupacional en el desempeño de sus tareas, percibiendo una cantidad adecuada de roles. Estos se ajustan a la rutina que ellos han organizado y que les permite desarroIlar una competencia ocupacional.

\section{Resultados en Mujeres}

- La noción del tiempo y los factores climáticos adquieren una importancia para el desarrollo de actividades, desempeño de roles y de rutina.

- Presentan una percepción de disponer de tiempo que les da la libertad de hacer las cosas con mayor tranquilidad, lo cual les permite concretar proyectos deseados.

- Identifican la necesidad de organizar de nuevo su vida y buscar un equilibrio en la manera de distribuir el tiempo. Dependiendo de la personalidad de cada una, aparece la necesidad de suplir la falta de trabajo realizando otras actividades de tipo productivo y de recreación tales como voluntariados, estudios, cursos, etc.

- "-Cuando en su situación actual de jubilada se presenta un evento de mucho significado, como un viaje al extranjero o visitas a sus hijos, tienen la posibilidad y la flexibilidad para postergar todos sus intereses, actividades y planificaciones futuras.

- La mayoría de las planificaciones son a corto plazo y no toman compromisos que no vayan más allá de un par de meses.

- El tiempo se vuelve indiferenciado, pasando a ser todos los días de la semana similares entre sí.

- Existe un prejuicio social de que el jubilado dispone de mucho tiempo para realizar cualquier tipo de actividad pero realmente se observa que no es así.

- Hay una sensación de pérdida en el sentido de pertenecer a una institución de trabajo y a un grupo de camaradas.

- Las jubiladas, cuya familia tiene una rutina estructurada, deben ajustarse a la dinámica familiar pudiendo disponer en un rango limitado de su tiempo, ya que deben ajustar sus actividades al funcionamiento y necesidades del resto, en contraposición a aquellas personas jubiladas que viven solas y que disponen de la libertad para organizar su tiempo entorno a las necesidades que se les presentan. 
- A pesar de que el descanso es una de las mayores motivaciones para jubilar, las proyecciones a futuro que presentan están vinculadas a realizar actividades que sean de su agrado, y esta sería la manera de descansar y no el estar "sin hacer nada".

\section{Conclusiones}

Es importante considerar para las conclusiones que el estudio presenta un sesgo en la muestra, que se encuentra relacionado con el nivel socioeconómico de los individuos que participaron de la investigación. Tales individuos pertenecen a un nivel socioeconómico medio-alto, por lo cual aquellos pertenecientes a un nivel socioeconómico medio-bajo podrían presentar un comportamiento diferente. Esto se debe principalmente a que los individuos que percibían menores ingresos económicos, continuaban desempeñando labores productivas, quedando excluidos al no cumplir con las características de la muestra.

Del análisis de los resultados obtenidos se desprende que -desde un enfoque Ocupacional- el proceso de jubilación no es percibido como negativo, lo cual se contrapone a la percepción actual que presentan otras disciplinas en relación a esta etapa de la vida.

Existen elementos recurrentes que surgen durante la investigación, los que avalan una percepción de satisfacción y adaptación a la nueva condición, los cuales se describen en detalle a continuación:

- Tanto en hombres como en mujeres se observa un cambio en el significado de las ocupaciones, ya que deben reemplazar la estructura de trabajo que antes tenían por otro tipo de actividades, de manera de no perder el equilibrio ocupacional que antes tenían.

- Se observa que el grupo de encuestados valora la importancia de mantener una rutina de estructura flexible que presente distintos tipos de actividades las cuales deben ser acordes a las necesidades que ellos presentan.

- En muchos casos, los encuestados manifiestan que al no encontrarse trabajando, la decisión de la distribución del tiempo es manejada por ellos y no condicionada por una estructura de trabajo. La estructura de trabajo era rígida y determinaba cuándo existía la posibilidad de realizar las obligaciones que no tuvieran relación con el trabajo, mientras que ahora, ya jubilados, pueden realizar las actividades en el momento que ellos estimen conveniente. Esto puede ser analizado desde un punto de vista negativo, ya que muchos lo describen como un "período de vacaciones permanente", perdiendo así el significado y valor que antes atribuían al disponer de tiempo libre.

En dos del total de entrevistados se observan características de disfunción ocupacional, lo cual coincide con que estas personas no están satisfaciendo sus necesidades ocupacionales, ya que desean retornar a sus labores productivas. Este deseo no cumplido interfiere en el desarrollo de todas las actividades que realiza a diario, entorpeciendo su Equilibrio Ocupacional.

Especialmente en el grupo de las mujeres, el hecho de ser dueñas de su propio tiempo es paradójico, ya que se comprometen con más demandas ocupacionales de manera de poder estructurar su vida cotidiana, en vez de disminuir el número de responsabilidades.

Es interesante destacar, que tanto los hombres como las mujeres entrevistadas tienen a sus padres vivos, y refieren el haber intensificado su rol de hijo al disponer ahora de mayor tiempo para dedicarle a sus padres.

Cabe destacar que muchos de los entrevistados coinciden con que tienen un ritmo de vida más lento, con menos estrés y mayor libertad para realizar las actividades que desean.

Otro factor recurrente dentro de este estudio es la falta de planes y proyectos futuros a largo plazo, destacándose planificaciones que no son proyectadas más allá de un mes.

De esta investigación también se desprende que la mantención de una rutina que satisface las necesidades individuales de cada uno, y que no necesariamente responde a la estructura propuesta por el $\mathrm{MOH}$, puede evitar una Disfunción Ocupacional y la posible aparición de una manifestación patológica. El $\mathrm{MOH}$ plantea que los ciclos desadaptativos generan un estado de disfunción ocupacional, cuando las expectativas sociales y las necesidades propias de exploración y dominio no son satisfechas ${ }^{233}$. Esta interacción es variable, ya que los individuos encuestados, no presentan una rutina socialmente aceptada, sin embargo no se 
observan características de disfunción ocupacional, existiendo una variación entre lo planteado por el $\mathrm{MOH}$ y lo que se observa en las rutinas que mantienen los individuos encuestados.

\section{Discusıón}

Cuando la rutina pasada se pierde, se observa un cambio en el significado y la valoración que tienen las antiguas ocupaciones así como también se aprecia una nueva valoración de las actuales ocupaciones. Esto concuerda con el estudio realizado por $\mathrm{H}$. Jonsson en el año $2000^{(22)}$, donde se plantea que las ocupaciones no tienen un significado por sí mismas, sino que cuando se encuentran en relación a otras ocupaciones y en la completa organización de la vida.

Los resultados obtenidos en el estudio coinciden con lo expresado en el marco teórico, en donde se plantea que la pérdida del trabajo produce una sensación de vacío en los individuos puesto que el trabajo sería el eje sobre el cual se desarrollan las otras ocupaciones, obligando al individuo a crear una nueva rutina con ocupaciones y sentidos significativos.

Por otra parte, los hallazgos de este estudio señalarían, que desde el punto de vista Ocupacional no se presenta, al menos en el grupo estudiado, una sensación de pérdida de capacidades para desempeñarse en los diversos roles, contradiciendo lo que plantea Domínguez ${ }^{(19)}$.

Del presente estudio se desprende que los roles se mantienen y que las capacidades para desempeñarlos se modifican de acuerdo a lo que los jubilados perciben como necesario de realizar, coincidiendo también con lo que plantea Aragoneses ${ }^{(5)}$ al referirse que las personas, al llegar a la etapa de jubilación, se desempeñan mayormente en actividades de tipo recreativas y de descanso.

Lo anteriormente expuesto se encuentra relacionado con lo planteado por el $\mathrm{MOH}$ donde la persona atraviesa una primera etapa de exploración, luego una de competencia y finalmente el logro, permaneciendo en aquellas actividades en donde experimentan mayor grado Los resultados arrojados en este estudio también respaldan el planteamiento hecho por George Maddos ${ }^{(5)}$ y el psiquiatra Francisco Alonso Fernández (11), los cuales se refieren a que un mayor número de roles o actividades ocupacionales y la organización son factores protectores y evitan un posible desequilibrio ocupacional. Esto se observa ya que trece de los quince entrevistados presentan estas características dentro de su desempeño ocupacional, mostrando un equilibrio ocupacional, mientras que los dos restantes que no cumplen con estas condiciones presentaban signos de una disfunción ocupacional.

Considerando lo planteado por Barros C. ${ }^{(21)}$, el presente estudio coincide con el planteamiento que la evaluación negativa acerca del retiro laboral se encuentra influenciada por la importante disminución de ingresos económicos, lo que provoca una limitación de la independencia y la posibilidad de realización personal.

Por otra parte, los resultados obtenidos no presentan concordancia con aquellos obtenidos por la misma autora, respecto de la disminución en las actividades domésticas por parte de los jubilados, ya que éstas se mantienen en el caso de las mujeres e incrementan en la totalidad de los encuestados hombres. Asimismo, cabe destacar que los jubilados que participan en este estudio presentan un aumento significativo de las actividades sociales, lo que es contrastante con los resultados obtenidos en el estudio realizado por Barros C. ${ }^{(21)}$, donde se plantea que las actividades disminuyen debido a pérdida de amistades por enfermedad, muerte, baja de ingresos económicos o temor a salir en la noche. Por otra parte existe una coincidencia en que hay una pérdida de contacto con aquellas personas con las que se interactuaba a diario en la situación de trabajo.

\section{REFLEXIÓN}

Este estudio pretende ser un aporte, desde la Ciencia de la Ocupación, para la formulación de Políticas de Jubilación para nuestra sociedad, las que deben orientarse a promover la salud y el bienestar a este grupo de individuos cuyo número enfrenta un gran aumento en este último tiempo.

Uno de los aportes más significativos de este estudio es que en su conjunto, avala los fundamentos filosóficos de la Terapia Ocupacional, expresados por la Asociación Americana de Terapia Ocupacional ${ }^{(4)}$ como lo siguientes: 
"El hombre es un ser activo, cuyo desarrollo está influenciado por el uso de la actividad propositiva. Utilizando su capacidad para la motivación intrínseca el ser humano es capaz de influir sobre su salud física y psíquica y sobre su entorno físico y social a través de la actividad propositiva. La vida humana es un proceso de continua adaptación. La adaptación es un cambio en la función que promueve la supervivencia y la puesta al día. Existen factores biológicos, psicológicos y ambientales que pueden interrumpir el proceso de adaptación en cualquier momento del ciclo vital, causando una disfunción. La actividad propositiva facilita el proceso adaptativo. La Terapia Ocupacional está basada en la creencia de que la actividad propositiva (ocupación), incluyendo sus componentes interpesonales y ambientales, puede utilizarse para prevenir y mejorar la disfunción y para promover el máximo nivel posible de adaptación."

\section{ReferenCias BibliográfiCAS}

Guzmán, J. M. (2002). Envejecimiento y Desarrollo en América Latina y el Caribe, Centro Latinoamericano y Caribeño de Demografía (CELADE) - División de Población; S/E; Santiago; Chile. Disponible en: www.eclac.cl/search/todositio.asp, (consultado en Marzo de 2004).

Quesney, F. (1988). Disfrute Plenamente Su Vejez. Santiago, Chile. Editorial La Puerta Abierta.

De Beauvoir, S. (1970). La Vejez. Buenos Aires. Argentina. Editorial Sudamericana. Segunda Edición.

Gómez, S., Rueda, L. (2002). Fundamento Teórico de la Terapia Ocupacional: Bases para el desarrollo de la ciencia de la ocupación. Universidad de Chile. Facultad de Medicina. Escuela de Terapia Ocupacional. Santiago, Chile. Texto Docente.

Padilla, V., Varela, M., Vega, C. (1994). Expectativas frente a la vida post jubilatoria, de trabajadores entre 60 y 64 años de edad, próximos a jubilar, de tres empresas del Área Metropolitana. Santiago, Chile. Memoria para optar al Título de Asistente Social, Licenciado en Trabajo Social.

Hopkins, H., Smith, H., Willard/Spackman Terapia Ocupacional. Madrid, España. Editorial Médica Panamericana. Octava Edición. 1998.

Diccionario Enciclopédico Planeta (1984). Tomo 6. Barcelona, España. Editorial Planeta. Primera Edición, 2785-6.

Valdés, M. A. (2000). Proyecto de Investigación para optar a Master en Integración de Persona con Discapacidad de la Universidad de Salamanca. Santiago, Chile. S/E .

Cox Edwards, A. (2000). El futuro de las pensiones en Chile: diferencias según sexo. Santiago, Chile. S/E. Disponible en: http:// www.cepchile.cl/dms/lang_1/doc_1606.html (consultado en abril de 2004).
Instituto de Normalización Previsional Ministerio del Trabajo y Previsión Social (1998). Guía Provisional del Adulto Mayor. Personal al servicio de las personas. Santiago, Chile. Publicación De La División De Asuntos Corporativos Del Instituto De Normalización Previsional. Tercera Edición.

Díaz Oviedo, C., Carrillo M. (2001). La prejubilación aumenta el riesgo de enfermedades cardiovasculares. Abril, 2001. Disponible en http://www.diariomedico.com/entorno/ent090401com.html (consultado en abril de 2004).

De Las Heras, C. G. (2004). “Organización Interna Del Sistema Humano Para La Ocupación”. Manual de Teoría Y Aplicación del Modelo de Ocupación Humana. Santiago, Chile. Capítulo 3.

De Las Heras, C. G. (2004). "Subsistema Volición".Manual de Teoría Y Aplicación del Modelo de Ocupación Humana. Santiago, Chile. Capítulo 4.

De Las Heras, C. G. (2004). "Subsistema Habituación".Manual de Teoría Y Aplicación del Modelo de Ocupación Humana. Santiago, Chile. Capítulo 5.

De Las Heras, C. G. (2004). “Subsistema Ejecución”. Manual de Teoría Y Aplicación del Modelo de Ocupación Humana. Santiago, Chile. Capítulo 6.

De Las Heras, C. G. (2004). "Influencias Ambientales En El Comportamiento Ocupacional".

Manual de Teoría Y Aplicación del Modelo de Ocupación Humana. Santiago, Chile. Capítulo 7. 2004.

Javet, L. (2004). "Sociodemografía. Impacto del Envejecimiento Poblacional". Apunte Asignatura Gerontología Social. Santiago, Chile. Escuela de Terapia Ocupacional, Universidad de Chile.

Moragas R. (2000). Preparación para la Jubilación. En: Fernández Ballesteros, R. Gerontología Social. Madrid-España: Ediciones Pirámide, pp. 453-471.

Domínguez O. (1982). La Vejez, Nueva Edad Social. Santiago, Chile: Editorial Andrés Bello.

Carrasco J. (2004). Máscaras y Expresión Corporal, un Vehículo al Teatro del Alma. Una Experiencia de la Unidad de rehabilitación del Instituto Psiquiátrico. Revista Chilena de Terapia Ocupacional. 4: 56-65.

Barros, C. (1991). Viviendo el Envejecer. Santiago, Chile. Instituto de Sociología, Pontificia Universidad católica de Chile, 67-72.

Jonsson H. (2000). Retirement: An Occupational Transition with Consequences on Temporality, Rhythm and Balance. Journal of Occupational Science. 7: 5-13.

Polonio B. y cols.- Conceptos Fundamentales de Terapia Ocupacional. Madrid, España. Editorial Médica Panamericana S.A. 2001.

Conceptos sobre Ocupación. Disponible en: http://www.walkredi.org/ PDF/Grupos\%20de\%20trabajo/CONCEPTOS\%20SOBRE\%20OC UPACION.d.pdf (consultado el 10 de Marzo de 2004). 